\title{
Khan-Malek anomaly-two cases of a never reported accessory coronary artery
}

\begin{abstract}
We report two cases of an unusual accessory coronary artery arising from the left coronary cusp and traveling over the superior epicardial aspect of the left ventricle parallel to the left main coronary (LM) and the left anterior descending artery (LAD).
\end{abstract}

Submitted: 18 August 2016; Accepted: 25 August 2016; Published online: 31 August 2016

Keywords: Coronary artery anomaly - Congenital coronary artery abnormality

We report two cases of an unusual accessory coronary artery arising from the left coronary cusp and traveling over the superior epicardial aspect of the left ventricle parallel to the left main coronary (LM) and the left anterior descending artery (LAD).

\section{Case 1}

A 54 year old male suffered an acute myocardial infarction and underwent successful percutaneous coronary intervention (PCI) with placement of a drug eluting stent (DES) to the LAD. He was discharged without complications and returned two weeks later for a staged PCI to the circumflex artery. Another DES was placed without event and the patient was discharged. Three months later he was screened and enrolled in an FDA approved stem cell trial for post myocardial infarction. During a planned angiogram a 5 French $\mathrm{XB} 3.0$ diagnostic catheter was advanced to the aortic root. In the left anterior oblique projection attempts at cannulation of the LM coronary artery proved difficult and the catheter tip lodged into a small vessel that arose from the left cusp. There was no damping of the catheter pressures and an angiogram was obtained using a hand injection (Figure 1).

The vessel was small in both size and distribution and had a noticeable ostial lesion.
Multiple projections and injections showed this vessel to originate in the left cusp near the LM and traveling over the high anterior wall of the left ventricle (Figure 2).

\section{Case 2}

A 62 year old male with history of hypertension presented with unstable angina, starting 1 day prior to presentation. Initial troponin was $<0.1 \mathrm{ng} / \mathrm{mL}$ and an exercise nuclear stress test demonstrated reversible ischemia with associated stress-induced diffuse ST depressions on the EKG portion of the study. The patient was then brought to the cardiac catheterization lab with findings of significant LM, LAD and circumflex disease, as well as incidental demonstration of an accessory coronary artery arising from the left coronary cusp and parallel to the LAD (Figure 3).

One injection resulted in enough reflux in the cusp to fill the LM coronary artery proving both its separate ostium and its proximity to the accessory artery (Figure 4). The patient was ultimately referred for bypass surgery.

\section{Discussion}

Anomalous coronaries occur in about $1 \%$ of the population and are usually benign and discovered as incidental findings during
Christina Tan, Hashim Khan, Mikhail Malek, Richard A Schatz*

Richard A Schatz, Scripps Clinic, La Jolla, San Diego CA, USA,

*Author for correspondence:

Tel.: 85855452487

Fax: +82422808238

RAS1024@aol.com 


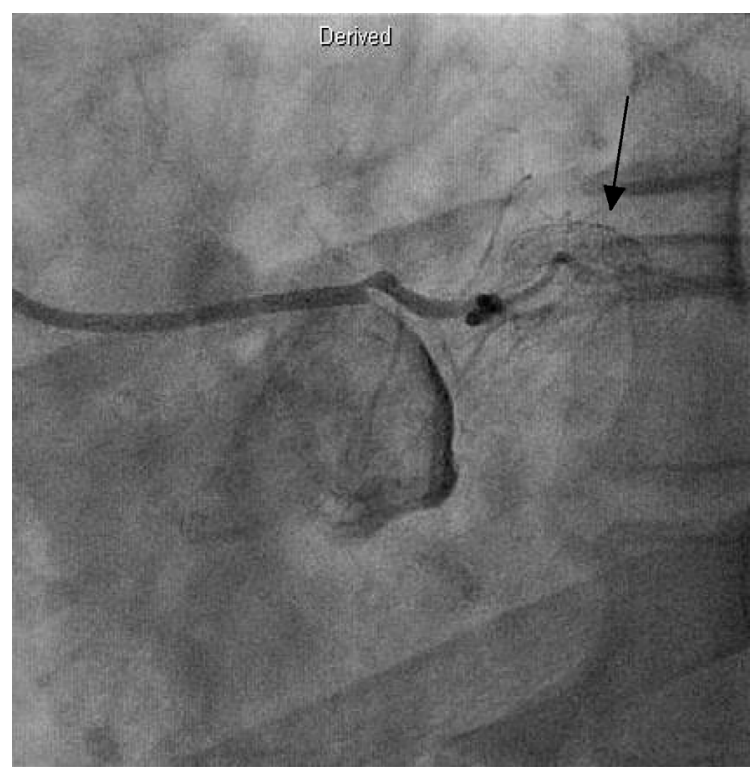

Figure 1. Injection of the left cusp reveals the accessory artery with ostial disease. The arrow points to previously placed stents in the LAD which shows that the accessory artery runs parallel to it.

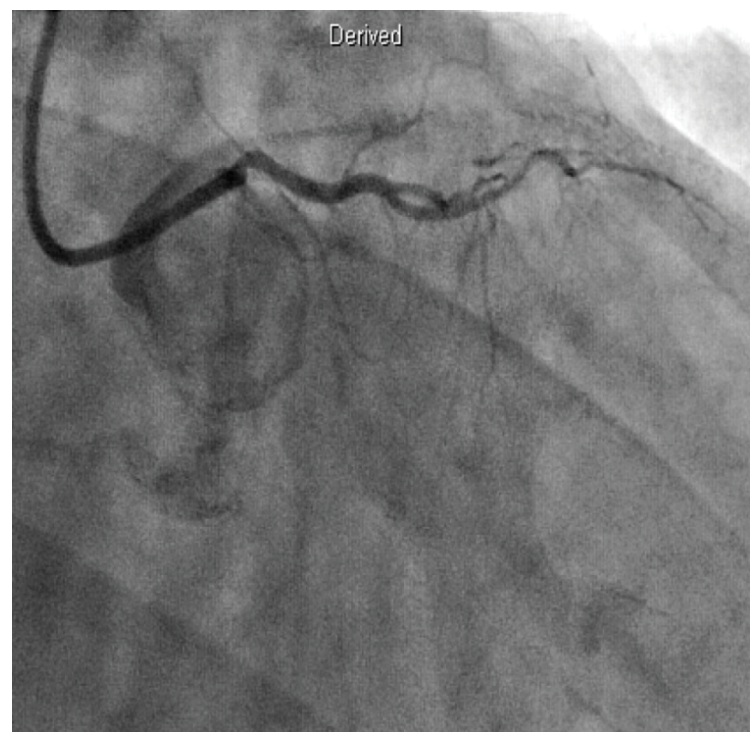

Figure 2. Right anterior oblique view of the accessory artery.

routine coronary angiography [1]. In a series of 1950 angiograms, the most common anomaly identified was a right coronary artery (RCA) arising from two separate ostia, which occurs in about $1.23 \%$ of identified anomalies, this was followed by an ectopic RCA from the right cusp in $1.13 \%$ and ectopic RCA from the left cusp in $0.92 \%$ [2]. In a series of 543 subjects studied with CT coronary angiography, the most common anomalies were absent left main in $3.3 \%$ of total subjects and $2.6 \%$ with rotation of the aortic root with normal coronary origins [3]. Particular anomalies, such as coronary artery origin from the wrong aortic sinus and coronary origin from the pulmonary trunk ("Bland-White-Garland Syndrome"), are associated with sudden cardiac death and surgical correction has often been performed in these cases [4].

Accessory arteries are rarely reported and represent additional arteries distinct from the usual anomalies classically described. In fact, in a large series representing screening of over 120,000 coronary angiograms, accessory arteries are not mentioned once confirming that they are strikingly rare [1]. CT coronary angiography may be better to define these unusual vessels; however the 2 large series to date totaling 1843 patients, accessory arteries are not mentioned [3]. Thus, it is reportable that two nearly identical accessory

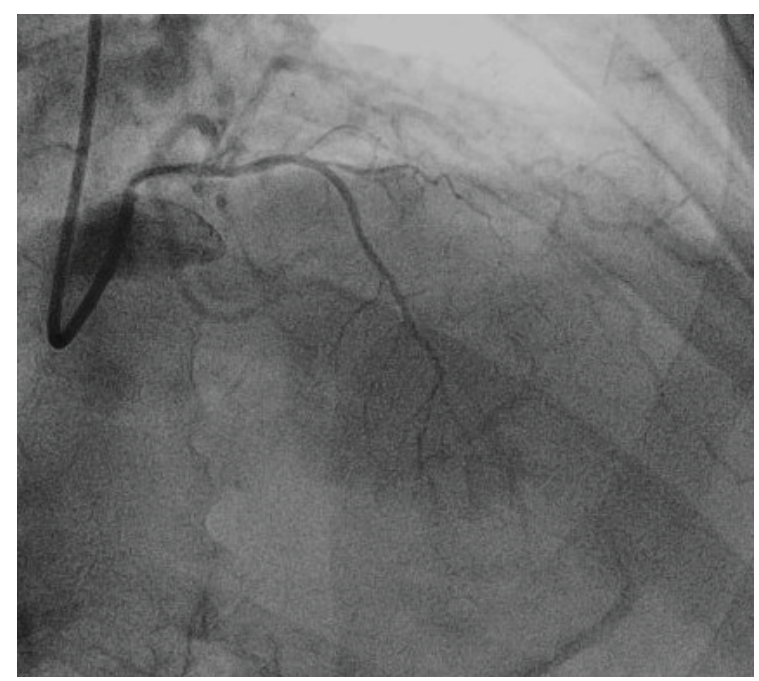

Figure 3. Right anterior oblique projection of the accessory artery.

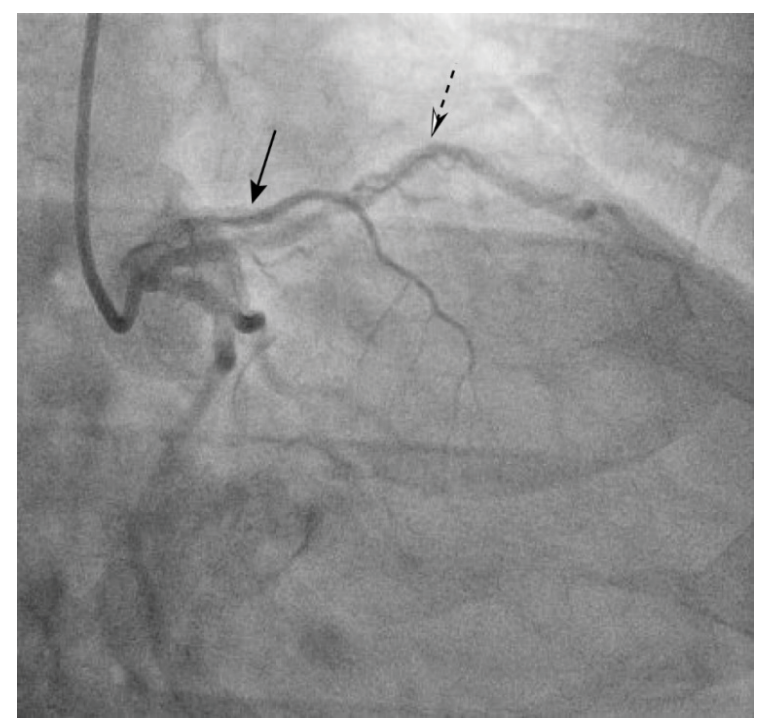

Figure 4. Left anterior oblique view of accessory artery (solid arrow) with contrast injection concurrently filling L main. The accessory artery (solid arrow) runs parallel to the LAD (dashed arrow) in its proximal course. 
arteries were discovered within weeks of each other in two different patients.

In neither of these cases were the arteries of any clinical significance despite one having an ostial lesion, presumably atherosclerotic in origin. Their size and distribution were nearly identical about $2 \mathrm{~mm}$ in diameter, running parallel to the left main coronary artery and terminating along the high anterior wall of the ventricle. It is presumed that they run posterior to the pulmonary artery as well due to the short distance travelled and absence of tortuosity.

Due to the striking rarity of this finding we propose the nomenclature "Khan-Malek Anomaly" after the trainee who discovered it and in memory of all trainees whose countless errant catheters passes usually draw the ire of the attending. Particularly relevant is the fact that several other experienced operators performed angiography on one of the patients and did not find this anomaly making it particular meaningful and fitting it be named after him. This is one time an errant catheter pass resulted in a significant finding.

\section{Executive summary}

- Accessory coronary arteries are an exceedingly rare and may be underdiagnosed due to difficulty identifying such vessels.

- In two cases of an accessory coronary artery parallel to the Left anterior descending artery, the prognosis associated with these findings was benign.

\section{References}

1. Yamanaka O, Hobbs RE. Coronary Artery Anomalies in 126, 595 Patients Undergoing Coronary Arteriography. Catheter. Cardiovasc. Diag. 21, 28-40 (1990).

2. Angelini P, Villason S, Chan AV, et al. Normal and anomalous coronary arteries in humans. In: Angelini P ed. Coronary Artery Anomalies: A Comprehensive Approach. Philadelphia, PA: Lippincott Williams \& Wilkins, 27-150 (1990).
3. Cademartiri F, La Grutta L, Malago R, et al. Prevalence of Anatomical Variants and Coronary Anomalies in 543 Consecutive Patients Studied with 64-slice CT Coronary Angiography. Eur. Radiol. 18, 781-791 (2008).

4. Basso C, Maron, BJ, Corrado D, et al. Clinical Profile of Congenital Coronary Artery Anomalies with Origin from the Wrong Aortic Sinus Leading to Sudden Death in Young Competitive Athletes. J. Am. Coll. Cardiol. 36, 1494-1501 (2000). 